\title{
Magnetically Driven Silver-Coated Nanocoils for Efficient Bacterial Contact Killing
}

\section{Journal Article}

\section{Author(s):}

Hoop, Marcus; Shen, Yang; Chen, Xiang-Zhong; Mushtaq, Fajer; luliano, Loredana M.; Sakar, Mahmut S.; Petruska, Andrew; Loessner, Martin J.; Nelson, Bradley J.; Pané, Salvador

\section{Publication date:}

2016-02-16

\section{Permanent link:}

https://doi.org/10.3929/ethz-b-000109585

\section{Rights / license:}

In Copyright - Non-Commercial Use Permitted

\section{Originally published in:}

Advanced Functional Materials 26(7), https://doi.org/10.1002/adfm.201504463

\section{Funding acknowledgement:}

336456 - Magnetoelectric chemonanorobotics for chemical and biomedical applications (EC) 
DOI: 10.1002/ ((please add manuscript number))

Article type: Full Paper

\section{Magnetically driven silver-coated nanocoils for efficient bacterial contact killing}

By Marcus Hoop, Yang Shen, Xiang-Zhong Chen*, Fajer Mushtaq, Loredana M. Iuliano, Mahmut S. Sakar, Andrew Petruska, Martin J. Loessner, Bradley J. Nelson, and Salvador Pané*.

M. Hoop, Dr. X.-Z. Chen, F. Mushtaq, Dr. M. S. Sakar, Dr. A. Petruska, Prof. Dr. B. J. Nelson, Dr. S. Pané

Institute of Robotics and Intelligent Systems (IRIS), ETH Zurich

Tannenstrasse 3, CH-8092 Zurich, Switzerland

E-mail: vidalp@ethz.ch, chenxian@ethz.ch

Dr. Y. Shen, Prof. Dr. M. J. Loessner

Institute of Food, Nutrition and Health, ETH Zurich

Schmelzbergstrasse 7, CH-8092 Zurich, Switzerland

M. Hoop and Dr. Y. Shen contributed equally to this research. 


\section{WILEY-VCH}

Keywords: Electrodeposition, nanocoil, magnetic, antibacterial effect, silver.

\section{Abstract}

The increasing threat of multidrug-resistant bacterial strains against conventional antibiotic therapies represents a significant worldwide health risk and intensifies the need for novel antibacterial treatments. In this work, an effective strategy to target and kill bacteria using silver-coated magnetic nanocoils is reported. The coil palladium (Pd) nanostructures are obtained by electrodeposition and selective dealloying, and subsequently coated with nickel (Ni) and silver (Ag) for magnetic manipulation and antibacterial properties, respectively. The efficiency of the nanocoils is tested in the treatment of Gram-negative Escherichia coli (E.coli) and Gram-positive methicillin-resistant Staphylococcus aureus (MRSA), both of which represent the leading multidrug-resistant bacterial pathogens. The nanocoils show highly effective bacterial killing activity at low concentrations and in relatively short durations of treatment time. Three different investigation techniques, LIVE/DEAD assay, Colony-Forming Unit (CFU) counting, and Scanning Electron Microscope (SEM), reveal that the antibacterial activity is a result of bacterial membrane damage caused by direct contact with the nanocoil. The low cytotoxicity towards fibroblast cells along with the capability of precise magnetic locomotion make the proposed nanocoil an ideal candidate to combat multidrug-resistant bacteria in the field of biomedical and environmental applications. 


\section{WILEY-VCH}

\section{Introduction}

The increasing emergence of multidrug-resistant pathogenic bacteria has gained recognition as a serious public health concern across the world. ${ }^{[1]}$ The Infectious Diseases Society of America estimates that over $70 \%$ of hospital-acquired infections are caused by multidrugresistant bacteria strains. ${ }^{[2]}$ Thus, the Center for Disease Control and Prevention declared the dawn of the "post antibiotic era". ${ }^{[3]}$ The major drawback of antibiotic therapy lies in its mode of inhibition of pivotal intracellular pathways ${ }^{[4]}$ instead of physically destroying the entire bacterium. ${ }^{[5,6]}$ This restricted effectiveness has been shown to foster the evolution of antibiotic resistant strains. ${ }^{[7]}$ To circumvent the problem of multidrug-resistance, new solutions that focus on high antibacterial efficiency by destroying the target microorganism are required. ${ }^{[8]}$ Additionally, conventional antibiotic treatments lack the ability to selectively target the site of bacterial infections. Therefore, novel strategies are required with targeted and destructive antibacterial activity against pathogenic bacteria, while minimizing the spread of multidrug-resistant pathogens and maintaining the natural host environment. ${ }^{[9]}$

Recently, developments in the emerging field of nanomedicine have generated promising strategies for the treatment of bacterial diseases caused by antibiotic-resistant strains. ${ }^{[10]}$ Such novel therapies comprise approaches such as bacteriophages ${ }^{[11]}$ and antibacterial polymers ${ }^{[5]}$. These therapies selectively kill bacteria by functionalizing the device with antibodies against specific bacterial strains. However, establishing these target domains is both highly laborious and limited by the identification of unique binding entities among various bacteria. ${ }^{[12]}$ In addition, most of these concepts still depend on existing antibiotic strategies and may not delay the onset of acquired resistance. ${ }^{[11]}$ A different approach in the field of nanomedicine is photothermal therapy, which utilizes hyperthermia reactions to effectively kill bacteria. ${ }^{[13-14]}$ It allows for externally controlled antibacterial activity through the use of near infrared laser light by exciting gold nanoparticles to induce localized hyperthermia even in deep tissues. ${ }^{[15]}$ But, the biomedical applicability of this treatment is limited by the risk of ablating vital cells 


\section{WILEY-VCH}

in the surrounding host environment. ${ }^{[16]}$ Alternatively, silver materials have been known since antiquity to exhibit a broad antibacterial spectrum combined with a reduced tendency to evoke microbial resistance due to its multiple target sites. ${ }^{[17]}$ Advances in antibacterial treatments at the nanoscale caused a revival of silver-based antiseptics. It has been demonstrated that silver increased the efficiency of antibiotics up to three orders of magnitude, while being effective against various bacterial species, such as Escherichia coli, Bacillus subtilis, and Staphylococcus aureus. ${ }^{[18-21]}$ Current research on antibacterial nano-agents is mainly focusing on silver nanoparticles, which have shown to exhibit inhibitory bacterial growth concentrations in the range of 10 to $20 \mu \mathrm{g} \mathrm{ml}^{-1}$ against $E$. coli depending on the size of the nanostructure. ${ }^{[22]}$ Furthermore, Wang et. al outlined the use of silver as a catalyst for selfpropelled micromotors. ${ }^{[23]}$ However, proposed strategies using silver nanostructures are still lacking adequate strategies for transport to the targeted bacterial infection site. Moreover, none of the above mentioned antibacterial approaches allow for post-treatment removal of the device.

We report for the first time, a novel approach towards targeted antibacterial therapy by fabricating silver-coated magnetic nanocoils for efficient bacteria killing. To generate swarms of nanocoils, we combined the fabrication of template-assisted electrodeposition and metal evaporation. Electrodeposition, as an inexpensive and versatile technique, allows for batch fabrication as well as precise control of geometrical features of the nanocoils.

Another advantage of the proposed nanocoil is that magnetic nanostructures can be precisely manipulated by corkscrew motion in all three dimensions, using low magnitude magnetic fields. ${ }^{[24]}$ Magnetic manipulation can be achieved by external systems that allow controlled locomotion in vivo (as previously demonstrated by our group ${ }^{[25]}$ ), enabling non-invasive, targeted delivery of the nanocoils to the place of bacterial infection as well as post-treatment removal. Antibacterial activity of the nanocoils was successfully demonstrated against representative Gram-negative E. coli as well as Gram-positive S. aureus strains, which are 


\section{WILEY-VCH}

known to cause life-threatening infections around the world. ${ }^{[26,27]}$ Furthermore, magnetic actuation experiments were conducted in order to demonstrate controlled locomotion of the nanocoils. The proposed nanocoil with its magnetic and antibacterial properties represents a novel biomedical and environmental approach for the treatment of antibiotic-resistant bacteria.

\section{Results and Discussion}

\subsection{Fabrication and characterization of nanocoils}

In this work, the coil Pd nanostructures were fabricated by template-assisted coelectrodeposition of Pd and $\mathrm{Cu}$ in anodized aluminum oxide (AAO) templates (Figure 1a (i)), as previously reported. ${ }^{[28]} \mathrm{Pd}$ nanocoils were obtained by means of selective etching of the AAO template (by $\mathrm{NaOH}$ ) and $\mathrm{Cu}$ segments (by $\mathrm{HNO}_{3}$ ) (Figure S1). To incorporate magnetic and antibacterial properties into the device, we covered the outer surface of the nanocoils with a uniform thin layer of $\mathrm{Ni}(7.5 \mathrm{~nm}$; Figure 1a (ii)) and Ag (10 nm; Figure 1a (iii)), respectively. Scanning Electron Microscopy (SEM) images (after each intermediate fabrication step) illustrate the changes in coil curvature (filament thickness Pd $-55 \mathrm{~nm}$; Pd/Ni - $64 \mathrm{~nm} ; \mathrm{Pd} / \mathrm{Ni} / \mathrm{Ag}$ - $73 \mathrm{~nm}$ ), as well as increased surface roughness after Ag-thin film evaporation. Energy dispersive X-ray spectrometry (EDX) was used to confirm the presence of Ni (14 at\%) and Ag (23 at\%) on the Pd (63 at\%) coil surface, by using a vector-based algorithm to determine the relative abundance of each element (Figure 1b). Furthermore, EDX mapping confirmed the uniform distribution of the Ag-layer on the Pd surface (Figure 1c-e).

\subsection{Antibacterial efficiency}

For the investigation of antibacterial activity of the Pd/Ni/Ag nanocoils, E. coli XL-1 blue MRF' cells were used as a Gram-negative bacteria for proof of concept. The killing efficiency of our nanocoil was quantified by counting colony-forming units (CFU; Figure S2). Based on previous investigations, the dominant antibacterial activity of our composite structure was 


\section{WILEY-VCH}

expected to be exhibited by the silver coating. ${ }^{[29-31]}$ To evaluate the antibacterial potential of our nanodevices from $\mathrm{Pd}^{[32]}$ and $\mathrm{Ni}^{[33]}$, nanocoils with $\mathrm{Pd}$ or $\mathrm{Pd} / \mathrm{Ni}$ were constructed for antibacterial testings. The results in Figure 2a show that the nanocoils exhibit significant bacterial killing effects on a log scale only when the outer layer of Ag is present. In fact, treatment with $100 \mathrm{ppm}\left[\mathrm{ppm}=\mu \mathrm{g} \mathrm{ml}^{-1}\right.$; corresponding to $23 \mu \mathrm{g} \mathrm{ml}^{-1}$ of Ag according to EDX analysis Figure 1b] was able to reduce bacterial viability within 2 h by six orders of magnitude, which corresponds to a 99.9999 \% killing efficiency. Nanocoils of $\mathrm{Pd}$ or Pd/Ni showed a decreased bacterial viability of $34 \%$ and $22 \%$, respectively. The dynamic killing activity was evaluated by CFU counting for $\mathrm{Pd} / \mathrm{Ni} / \mathrm{Ag}$ nanocoils in the concentration range of 5-100 ppm at various time points $(0,1,2,4$, and 6 h). Figure $2 \mathrm{~b}$ shows a dose-dependent killing with a minimum bactericidal concentration $(\mathrm{MBC})^{[34]}$ of the Ag-covered nanocoils at $30 \mathrm{ppm}$. At low concentration, 5 and $10 \mathrm{ppm}$ of nanocoils were able to inhibit the growth of bacteria after $2 \mathrm{~h}$ of incubation, compared to the control group (0 ppm).

The geometrical properties of nanostructures have been demonstrated to play an important role for the antibacterial efficiency of Ag-nanodevices (Table S1). ${ }^{[35]}$ In order to elucidate the impact of the coil shape and surface roughness on the killing efficiency, we compared the antibacterial properties of $\mathrm{Pd}$ and $\mathrm{Pd} / \mathrm{Ni} / \mathrm{Ag}$ nanocoils with $\mathrm{Pd}$ and $\mathrm{Pd} / \mathrm{Ni} / \mathrm{Ag}$ nanowires of the same size. Figure S3 shows that nanocoils with solely Pd was able to reduce the bacterial viability by $34 \%$. In comparison, Pd nanowires of the same size reduced bacterial viability by only $6 \%$. These results indicate that the coil like feature of our nanostructure alone contributes to the bacterial killing efficiency. However, the difference in antibacterial properties depending on shape was reduced upon introducing Ag as an antibacterial agent at a higher concentration of $10 \mathrm{ppm}$. In that case, no significant difference between the nanocoils and nanowires were observed and the main antibacterial properties are likely attributed to the Ag coating. 


\section{WILEY-VCH}

Next, a LIVE/DEAD BacLight staining assay was performed after E.coli were incubated with

50 ppm Pd/Ni/Ag nanocoils. The two fluorescent dyes, SYTO9 and propidium iodide (PI), allow discrimination of intact (green) and damaged (orange-red) bacterial membranes, respectively. ${ }^{[36]}$ Analysis by CLSM confirmed the time-dependent induction of bacterial killing (Figure 2c-d). Interestingly, the shape of the bacteria remained intact even though the PI staining indicated a compromised bacterial membrane. The results suggest that the Agcoated nanocoils induce membrane disintegration as the antibacterial mode of action.

\subsection{Bacterial killing mechanism}

Despite efforts in the literature to investigate the the bacterial killing mechanism of silver, the exact mechanism remains unclear. ${ }^{[37,38]}$ Although several publications argue that the dissolution of Ag in the form of Ag-ions is the main cause for the antibacterial efficiency, ${ }^{[19}$, ${ }^{39]}$ more severe antibacterial effects through membrane damage have been attributed to ionic Ag on the surface of nanoparticles. ${ }^{[35]}$ In this context, Lok and co-workers concluded that bacterial toxicity of Ag nanoparticles is three orders of magnitude higher than that of Ag-ions at the subnanomolar concentration. ${ }^{[40]}$ To test whether the dissolution of Ag-ions is the primary cause for the antibacterial effect, we performed Ag-ion dissolution tests from our $\mathrm{Pd} / \mathrm{Ni} / \mathrm{Ag}$ nanocoils as control experiments. ${ }^{[41]}$ Ag-coated nanocoils at a concentration of 100 ppm were stored for two days in DI water. Nanocoils were collected with a magnet and the supernatant was tested for antibacterial effects. Figure S4 shows no significant bacteria killing effect caused by the supernatant in comparison to the Ag-coated nanocoils. Based on this result, we conclude that a potential dissolution of Ag-ions from our structures is not the dominant cause for the bacteria killing effect. SEM analysis of E.coli after antibacterial treatment further revealed significant surface morphology changes upon interaction with $\mathrm{Pd} / \mathrm{Ni} / \mathrm{Ag}$ nanocoils, whereas bacteria not in contact with the nanocoil maintained their regular surface morphology (Figure 3a-b). Hence, we speculate that the bacteria killing mechanism of the nanocoils is initiated by membrane disintegration caused by direct contact 


\section{WILEY-VCH}

interaction with the Ag-coating on the surface of the nanocoil and is not mediated by the release of Ag-ions.

The hypothesis of a 'direct contact killing mode' was further corroborated by antibacterial tests (Figure 3c, Figure S5) on Ag-ion-tolerant (laboratory-adapted E.coli strain which tolerates silver nitrate concentration up to $320 \mu \mathrm{g} \mathrm{ml}{ }^{-1}$, unpublished data) and Ag-ionsensitive E.coli strains. In both cases, the bacterial killing efficiency of our Pd/Ni/Ag nanocoil was comparable to the results obtained from the E.coli XL-1 blue cells. Hence, the direct contact between bacteria and Ag surface of the nanocoils is assumed to be the primary cause for the bacteria killing effect.

Further reports in the literature argue that the antibacterial activity of Ag could also be a result of the generation of reactive oxygen species (ROS). ROS are short living and highly reactive oxidants, which are the main killing mechanism used by many antibacterial drugs as well as antibiotics. ${ }^{[42]}$ To investigate the influence of ROS on the antibacterial mechanism of our Pd/Ni/Ag nanocoils, E.coli XL 1 cells were incubated with different concentrations of the nanocoils (0-100 ppm) under anaerobic condition, which is known to prevent ROS generation due to the a lack of dissolved oxygen. ${ }^{[4]}$ Figure S5 shows similar killing efficiency of the $\mathrm{Pd} / \mathrm{Ni} / \mathrm{Ag}$ nanocoils under anaerobic conditions. Hence, our results suggest that the generation of ROS is not the main cause for the antibacterial mechanism of our proposed nanostructures. Although the antibacterial activity of Ag-based nanostructures has been documented in the literature, their efficiency against Gram-positive pathogens was limited (less than 1 logarithm unit or $40 \%$ reduction), possibly due to the protection of the outer peptidoglycan layer or peptidoglycan-associated polymers. ${ }^{[44]}$ Strikingly, with the proposed novel nanocoil structure, we were able to achieve significant antibacterial efficiency of 2 logarithm units (99\% reduction in viability) at $100 \mathrm{ppm}$ within $2 \mathrm{~h}$ against clinically-relevant MRSA. The effective killing of multidrug resistant bacterial strains highlights the potential of our nanocoil to be an ideal tool to circumvent drawbacks of traditional antibiotic treatment strategies. 


\section{WILEY-VCH}

The results of this work show that the antibacterial effect of our nanocoil is primarily caused by direct interaction. In fact, the high bacterial killing activity against Ag-ion tolerant E. coli, the low antibacterial activity of the nanocoil supernatant, and the high antibacterial efficiency under anaerobic conditions suggest that the main mode of action is not caused by the release of Ag-ions or generation of ROS, but rather mediated by direct interaction with the Ag surface. Silver is known to have a tendency to react with phosphorous containing chemical entities. ${ }^{[45,46]}$ Thus, phosphorous-containing lipopolysaccharides (Gram-negative bacteria) ${ }^{[47]}$ and teichoic acids (Gram-positive bacteria) ${ }^{[48]}$ on the outer bacterial surface are susceptible to silver, potentially causing the observed surface morphology changes. Furthermore, our experimental data suggest that the antibacterial efficiency of our nanocoil at low concentrations can be further attributed to its nanosized shape with high surface roughness, which has also been shown to exert mechanical stress on the membrane for efficient bacteria killing by other nanodevices. ${ }^{[49,50]}$

\subsection{Cytotoxicity and magnetic actuation}

In order to demonstrate the potential of our proposed antibacterial nanocoil as a tool for biomedical and environmental applications, we tested the cytotoxicity of the $\mathrm{Pd} / \mathrm{Ni} / \mathrm{Ag}$ nanocoils with mouse fibroblast NIH-3T3 cells by MTT assay, a methodology used before for evaluating the biocompatibility for nanomotors by Chng et. al. ${ }^{[51]}$ In agreement with the literature, Figure 4a shows that the silver content of our structures even at the highest concentration of $100 \mathrm{ppm}$ is below the threshold to exhibit toxicity towards the tested NIH3T3 cells after 24 h treatment. ${ }^{[52]}$ This result demonstrates that the killing efficiency of the nanocoil is only directed towards bacteria, which is imperative for its potential in biomedical application .

Besides the efficiency of silver-based antibacterial nanocoils, applications in the biomedical field make it compulsory that novel strategies are applied to specifically target the bacteria site and enable the recovery of the device after treatment. This can be accomplished by using 


\section{WILEY-VCH}

nanostructures with incorporated motion control. ${ }^{[53]}$ This feature was tested by controlled propulsion experiments of our $\mathrm{Pd} / \mathrm{Ni} / \mathrm{Ag}$ coated nanocoils using uniform low-magnitude magnetic fields, which allow externally controlled locomotion of nanodevices even in vivo. ${ }^{[25,}$ 54, 55] In order to demonstrate this magnetic locomotion of our proposed nanocoil, the structures were dispersed in DI water and magnetic fields were applied. Time-lapse images shown in Figure 4b illustrate the trajectory of the magnetic nanocoils for a defined motion over a 3 s time period (Supplementary Video).

\section{Conclusions}

In this work, we demonstrate the fabrication of magnetic nanocoils with antibacterial properties by using electrodeposition, selective etching and metal evaporation techniques. This is the first work that reports magnetic nanocoils with antibacterial properties. The advantage of our nanocoil, over current state-of-the-art nanoparticle approaches, is the ability to perform external magnetic manipulation even at low magnetic fields. This allows for controlled locomotion towards the infection area as well as post-treatment recovery without harming the surrounding environment, as required in biomedical and environmental applications. The high effectiveness of the nanocoils against Gram-positive and Gramnegative bacteria strains was attributed to a contact killing mechanism, which aids to circumvent antibiotic resistance. Furthermore, it was shown that the coil shape contributes to the antibacterial properties, particularly at low concentrations. The results of this work encourage the application of our nanocoils in the field of biomedicine, water and air purification, cosmetics and food against various other pathogenic, and multidrug-resistant bacteria.

\section{Experimental Section}

\subsection{Fabrication of magnetic, antibacterial nanocoils}




\section{WILEY-VCH}

Electrodeposition of nanocoils was conducted in commercial anodized aluminum oxide (AAO) templates (Whatman ${ }^{\circledR}$ ) with pore sizes of $200 \mathrm{~nm}$. Prior to deposition, a $100 \mathrm{~nm}$ thick layer of Au was evaporated by electron beam evaporation on the backside of the AAO template, serving as a conductive working electrode. First, Au nanorods ( $2 \mu \mathrm{m})$ were plated from a gold potassium cyanide plating solution ( $\mathrm{pH}$ 3.8) at $-2 \mathrm{~mA} \mathrm{~cm}^{-2}$ in a two-electrode system with a platinized titanium counter electrode. All further electrochemical deposition experiments were conducted with an Autolab PGSTAT302N potentiostatic in a threeelectrode cell with platinum sheet and $\mathrm{Ag} / \mathrm{AgCl}$ (with $1 \mathrm{M} \mathrm{KCl}$ ) as the counter and reference electrode, respectively. Next, $\mathrm{Pd} / \mathrm{Cu}$ nanorods were fabricated at room temperature and with an applied constant voltage of $-0.095 \mathrm{~V}$ from a $30 \mathrm{mM} \mathrm{PdCl}_{2}$ and $20 \mathrm{mM} \mathrm{CuCl}_{2}$ (pH 1.3) plating solution with constant stirring. Afterwards, the gold, AAO template and $\mathrm{Cu}$ were removed by commercially available gold etchant solution (GE-8148, Transene), $5 \mathrm{M} \mathrm{NaOH}$ and $8 \mathrm{M} \mathrm{HNO}_{3}$, respectively. Obtained Pd nanocoils were washed in ultrapure water and collected by centrifugation until neutral pH was reached. Finally, 7 and $10 \mathrm{~nm}$ thick layers of $\mathrm{Ni}$ and Ag were deposited by physical vapor deposition on Pd nanocoils, respectively. The morphology, after each fabrication step, was investigated by SEM (Zeiss Ultra) operating at $5 \mathrm{kV}$. Furthermore, the chemical composition of the Pd nanocoil and the presence of the $\mathrm{Ni}$ and Ag layer were investigated by energy dispersive X-ray analysis (EDX, FEI Quanta200). For SEM and EDX preparation, the respective nanocoil samples in DI water were sonicated for 2 min. Afterwards the solution containing dispersed nanocoils was dropped on a conductive silicon chip and the liquid was evaporated by air-drying.

$\mathrm{Pd}, \mathrm{Pd} / \mathrm{Ni} / \mathrm{Ag}$ nanowires were fabricated as described above for nanocoils. Differences only applied to the electrolyte composition (30 $\mathrm{mM} \mathrm{PdCl}_{2}$ at $\mathrm{pH} 1.3$ ).

\subsection{Assessment of antibacterial activity of $\mathrm{Pd} / \mathrm{Ni} / \mathrm{Ag}$ nanocoils}

E.coli strain XL1-blue MRF' cells (Stratagene), Ag-sensitive E.coli ATCC8739 (DSM 1576), and Ag-tolerant E.coli (laboratory adapted E.coli ATCC8739 to up to $320 \mu \mathrm{g} \mathrm{ml}^{-1}$ 


\section{WILEY-VCH}

silver nitrate) were used for the assessment of antibacterial activity of the $\mathrm{Pd} / \mathrm{Ni} / \mathrm{Ag}$ nanocoils.

The adaption of E.coli to silver ion was performed in the laboratory by step-wise increment with sub-lethal concentrations for 16 generations. The minimum inhibitory concentration of silver nitrate against Ag-sensitive E.coli ATCC8739 is $4 \mu \mathrm{g} \mathrm{ml}^{-1}$ in LB medium. In the end, the adapted E. coli was shown to tolerate up to $320 \mu \mathrm{g} \mathrm{ml}^{-1}$ of silver nitrate (unpublished data). The bacteria was inoculated and grown in Lysogeny Broth (LB) media overnight under aerobic conditions. Clinically-isolated methicillin-resistant Staphylococcus aureus ZH124 (University Zurich, Zurich, Switzerland) was grown in Brain Heart Infusion (BHI) media overnight prior to antimicrobial tests. Bacterial density was determined by $\mathrm{OD}_{600}$ measurements and adjusted to approximately $10^{8}$ bacterial CFU ml ${ }^{-1}$ for E.coli and $10^{6} \mathrm{CFU}$ $\mathrm{ml}^{-1}$ for MRSA with broth media, respectively. $50 \mathrm{ppm}$ of the $\mathrm{Pd} / \mathrm{Ni} / \mathrm{Ag}$ nanocoils were incubated with bacterial cells at $37^{\circ} \mathrm{C}$. After 1-6 h, the magnetic coils were collected with a magnet and $100 \mu \mathrm{L}$ of the bacterial suspension was distributed on an LB agar plate (for E.coli) and BHI agar plate (for MRSA) at different dilutions. The plates were further incubated at $37{ }^{\circ} \mathrm{C}$ for $24 \mathrm{~h}$ and bacteria viability was determined by counting the colony forming units (CFU). A second aliquot of the incubated bacteria and nanocoils suspension was used for LIVE/DEAD BacLight viability assay (Life Technology). Here, the nanocoils were removed by a magnet and the bacteria suspension was incubated for 10 min with propidium iodide and SYTO9 according to the manufacturer's instruction. The viability of the bacteria cells was assessed by Leica TCS SPE confocal microscope at wavelength of 480/500 nm (SYTO9) and 490/635 nm (PI) (Leica Microsystems GmbH, Wetzlar, Germany), operated by Leica LAS AF interface.

To investigate possible antibacterial effects of released $\mathrm{Ag}$ ions, the $\mathrm{Pd} / \mathrm{Ni} / \mathrm{Ag}$ nanocoils (100 ppm) were incubated for $48 \mathrm{~h}$, collected with a magnet, and the supernatant was used for the antibacterial assay depicted above, followed by CFU counting on $24 \mathrm{~h}$ incubated agar plates. 


\section{WILEY-VCH}

In order to test the effect of ROS species on the killing mechanism of the $\mathrm{Pd} / \mathrm{Ni} / \mathrm{Ag}$ nanocoils, CFU counting experiments of E.coli XL 1 cells were conducted as described above, yet by anaerobic conditions under an anaerobic cell culture bench.

The influence of nanocoil shape on the killing efficiency of the nanodevices was investigated by CFU counting experiments of E.coli XL 1 cells incubated with Pd nanocoils and -wires (50 ppm) as well as $\mathrm{Pd} / \mathrm{Ni} / \mathrm{Ag}$ nanocoils and -wires (0-10 ppm).

Time and concentration dependency of the antibacterial effect was studied by conducting the antibacterial assay with different concentrations of the $\mathrm{Pd} / \mathrm{Ni} / \mathrm{Ag}$ nanocoils $(0-100 \mathrm{ppm})$. Aliquots of the bacteria/ nanocoils samples were taken at each time point and used for serial dilution on agar plates. Quantification of the viability was conducted after 24 h by CFU count. The morphological changes on the bacteria surface upon contact with the Ag-coated nanocoil nanostructures was studied under the SEM (Zeiss Ultra). Nanocoils and E.coli were incubated for $1 \mathrm{~h}$. Silicon wafer were treated with $0.01 \%$ poly-L-lysine for 10 min and dried at room temperature. Then, bacteria/ nanocoil solution was dropped on the silicon wafer and attachment on the poly-L-lysine was achieved by incubation at $37^{\circ} \mathrm{C}$. Afterwards, chips were washed in PBS and placed 15 min at room temperature in 2.5\% Glutaraldehyde/PBS solution. After three washing steps in PBS, post fixation of the sample was done in $1 \% \mathrm{OsO}_{4} / \mathrm{PBS}$, followed by additional washing steps in PBS. Dehydration of the sample was achieved by placing the chips in 30\% Ethanol (5 min), 50/70/90\% Ethanol (10 min), and three times in 100\% Ethanol for 10 min. Next, samples were used for Critical Point Drying (Tousimis 931 GL) with $\mathrm{CO}_{2}$. For SEM imaging the bacteria were coated with a $3 \mathrm{~nm}$ platinum layer by sputter coating.

\subsection{Cell Viability}

The mouse fibroblast NIH-3T3 cells were cultivated in cell culture medium (DMEM, 10\% FCS; 100 x Antimycoticum) at physiological conditions. The MTT Cytotoxicity study was conducted in 96-well plates with $1 \times 10^{4} 3 \mathrm{~T} 3$ cells in culture medium (100 $\left.\mu \mathrm{L}\right)$. Cells were 


\section{WILEY-VCH}

allowed to attach for $4 \mathrm{~h}$, cell medium was removed, cells were washed with PBS and exposed to different concentrations of the $\mathrm{Pd} / \mathrm{Ni} / \mathrm{Ag}$ nanocoils. After $24 \mathrm{~h}$ of incubation, the supernatant was replaced by fresh media $(100 \mu \mathrm{L})$ and supplemented with MTT (12 mM). After $4 \mathrm{~h}$ of incubation, isopropanol $(100 \mu \mathrm{L})$ and $\mathrm{HCl}(0.04 \mathrm{M})$ were added to the cells. Absorbance measurements were conducted in a micro-titer plate reader (Infinite f200 Tecan) at $540 \mathrm{~nm}$.

\subsection{Magnetic Actuation}

The nanocoils were manipulated by a customized magnetic actuation system (MFG-100-I, MagnetbotiX AG, Switzerland). The rotating magnetic fields were generated by eight opposing coils $(8 \mathrm{mT} ; 10 \mathrm{~Hz})$. The $\mathrm{Pd} / \mathrm{Ni} / \mathrm{Ag}$ nanocoils were dispersed in DI water, placed underneath the coil system and imaged with an integrated inverted optical microscope (Olympus IX-81).

\section{Supporting Information}

Supporting Information is available from the Wiley Online Library or from the author.

\section{Acknowledgements}

This work has been financed by the European Research Council Starting Grant "Magnetoelectric Chemonanocoilics for Chemical and Biomedical Applications (ELECTROCHEMBOTS)”, by the ERC grant agreement no. 336456. We thank Prof. Dr. Brigitte Berger-Bächi at University Zurich for providing the isolated methicillin-resistant Staphylococcus aureus strains. We also thank Dr. Mathias Schmelcher for his critical comments. The authors would like to acknowledge the Scientific Center for Optical and Electron Microscopy (ScopeM) of ETH, and the FIRST laboratory for their technical support.

Received: ((will be filled in by the editorial staff))

Revised: ((will be filled in by the editorial staff)) Published online: ((will be filled in by the editorial staff)) 


\section{References}

[1] J. Davies, D. Davies, Microbiol. Mol. Biol. Rev. 2010, 74, 417.

[2] A. E. Clatworthy, E. Pierson, D. T. Hung, Nat. Chem. Biol. 2007, 3, 541.

[3] CDC. Antibiotic resistance threats in the United States 2013. http://www.cdc.gov/drugresistance/threat-report-2013/pdf/ar-threats-2013-508.pdf （accessed May 14, 2015).

[4] M. A. Kohanski, D. J. Dwyer, J. J. Collins, Nat. Rev. Microbiol. 2010, 8, 423.

[5] F. Nederberg, Y. Zhang, J. P. Tan, K. Xu, H. Wang, C. Yang, S. Gao, X. D. Guo, K. Fukushima, L. Li, J. L. Hedrick, Y. Y. Yang, Nat. Chem. 2011, 3, 409.

[6] A. J. Friedman, J. Phan, D. O. Schairer, J. Champer, M. Qin, A. Pirouz, K. BlecherPaz, A. Oren, P. T. Liu, R. L. Modlin, J. Kim, J. Invest. Dermatol. 2013, 133, 1231.

[7] S. B. Levy, B. Marshall, Nat. Med. 2004, 10, S122.

[8] L. L. Silver, Nat. Rev. Drug Disc. 2007, 6, 41.

[9] H. Loferer, Mol. Med. Today 2000, 6, 470ma.

[10] I. Yacoby, I. Benhar, Nanomedicine 2008, 3, 329.

[11] I. Yacoby, H. Bar, I. Benhar, Antimicrob. Agents Chemother. 2007, 51, 2156.

[12] I. Yacoby, I. Benhar, Infect. Disord. Drug Targets 2007, 7, 221.

[13] N. J. Millenbaugh, J. B. Baskin, M. N. DeSilva, W. R. Elliott, R. D. Glickman, Int. J. Nanomed. 2015, 10, 1953.

[14] W. C. Huang, P. J. Tsai, Y. C. Chen, Nanomedicine 2007, 2, 777.

[15] Z. Fan, X. Dai, Y. Lu, E. Yu, N. Brahmbatt, N. Carter, C. Tchouwou, A. K. Singh, Y. Jones, H. Yu, P. C. Ray, Mol. Pharm. 2014, 11, 1109.

[16] J. R. Melamed, R. S. Edelstein, E. S. Day, ACS Nano 2015, 9, 6.

[17] M. Rai, A. Yadav, A. Gade, Biotechnol. Adv. 2009, 27, 76. 


\section{WILEY-VCH}

[18] G. Zhao, S. E. Stevens, Biometals 1998, 11, 27.

[19] C. Marambio-Jones, E. M. Hoek, J. Nanopart. Res. 2010, 12, 1531.

[20] J. R. Morones-Ramirez, J. A. Winkler, C. S. Spina, J. J. Collins, Sci. Transl. Med. 2013, 5, 190 ra181.

[21] A. R. Shahverdi, A. Fakhimi, H. R. Shahverdi, S. Minaian, Nanomedicine 2007, 3, 168.

[22] S. Chernousova, M. Epple, Ang. Chem. Int. Ed. 2013, 52, 1636.

[23] H. Wang, G. Zhao, M. Pumera, J. Am. Chem. Soc. 2014, 136, 2719.

[24] Y. F. Shen, Robotics Biomim. 2014, 1, 1.

[25] A. Servant, F. Qiu, M. Mazza, K. Kostarelos, B. J. Nelson, Adv. Mater. 2015, 27, 2981.

[26] M. Z. David, R. S. Daum, Clin. Microbiol. Rev. 2010, 23, 616.

[27] A. Waness, J. Glob. Infect. Dis. 2010, 2, 49.

[28] a) J. X. Li, S. Sattayasamitsathit, R. F. Dong, W. Gao, R. Tam, X. M. Feng, S. Ai, J. Wang, Nanoscale 2014, 6, 9415. b) L. C. Liu, S. H. Yoo, S. A. Lee, S. H. Park, Nano Lett. 2011, 11, 3979.

[29] C. K. Melber, I. Mangelsdorf, Geneva: World Health Organization; 2002.

[30] D. H. Nies, Appl. Microbiol. Biotechnol. 1999, 51, 730.

[31] J. Li, S. X. Lin, J. Wang, S. Jia, M. Y. Yang, Z. Y. Hao, X. Y. Zhang, P. R. Chen, J. Am. Chem. Soc. 2013, 135, 7330.

[32] C. P. Adams, K. A. Walker, S. O. Obare, K. M. Docherty, PLoS One 2014, 9 (1). e85981.

[33] M. Yasuyuki, K. Kunihiro, S. Kurissery, N. Kanavillil, Y. Sato, Y. Kikuchi, Biofouling 2010, 26 (7), 851.

[34] R. Jones, A. Barry, T. Gavan, American Society for Microbiology: Washington, 1985; 972. 


\section{WILEY-VCH}

[35] S. Pal, Y. K. Tak, J. M. Song, Appl. Environ. Microbiol. 2007, 73 (6), 1712.

[36] M. Berney, F. Hammes, F. Bosshard, H. U. Weilenmann, T. Egli, Environ. Microbiol. 2007, 73, 3283.

[37] K. B. Holt, A. J. Bard, Biochemistry 2005, 44, 13214.

[38] W. R. Li, X. B. Xie, Q. S. Shi, H. Y. Zeng, Y. S. Ou-Yang, Y. B. Chen, Appl. Microbiol. Biotechnol. 2010, 85, 1115.

[39] S. Kittler, C. Greulich, J. Diendorf, M. Koller, M. Epple, Chem. Mater. 2010, 22, 4548.

[40] C. N. Lok, C. M. Ho, R. Chen, Q. Y. He, W. Y. Yu, H. Sun, P. K. Tam, J. F. Chiu, C. M. Che, J. Proteome Res. 2006, 5, 916.

[41] K. Loza, J. Diendorf, C. Sengstock, L. Ruiz-Gonzalez, J. M. Gonzalez-Calbet, M. Vallet-Regi, M. Koller, M. Epple, J. Mater. Chem. B 2014, 2, 1634.

[42] M. A. Kohanski, D. J. Dwyer, B. Hayete, C. A. Lawrence, J. J. A. Collins, Cell 2007, 130 (5), 797.

[43] W. W. He, H. M. Jia, D. F. Yang, P. Xiao, X. L. Fan, Z. Zheng, H. K. Kim, W. G. Warner, J. J. Yin, ACS Appl. Mater. Interfaces 2015, 7 (30), 16440.

[44] M. Mahmoudi, V. Serpooshan, ACS Nano 2012, 6, 2656.

[45] J. R. Morones, J. L. Elechiguerra, A. Camacho, K. Holt, J. B. Kouri, J. T. Ramirez, M. J. Yacaman, Nanotechnology 2005, 16, 2346.

[46] S. J. Brokx, M. Ellison, T. Locke, D. Bottorff, L. Frost, J. H. Weiner, J. Bacteriol. 2004, 186, 3254.

[47] P. O. Magalhaes, A. M. Lopes, P. G. Mazzola, C. Rangel-Yagui, T. C. Penna, Jr. A. Pessoa, J. Pharm. Pharm. Sci. 2007, 10, 388.

[48] M. Gross, S. E. Cramton, F. Gotz, A. Peschel, Infect. Immun. 2001, 69, 3423.

[49] S. Liu, L. Wei, L. Hao, N. Fang, M. W. Chang, R. Xu, Y. Yang, Y. Chen, ACS Nano 2009, 3, 3891. 


\section{WILEY-VCH}

[50] O. Akhavan, E. Ghaderi, ACS Nano 2010, 4, 5731.

[51] E. L. K. Chng, G. Zhao, M. Pumera, Nanoscale 2014, 6, 2119.

[52] I. Sur, D. Cam, M. Kahrmann, A. Baysal, M. Culha, Nanotech. 2010, 21, 175104.

[53] A. J. Cozzone, Emerg. Microbes Infec. 2012, 1, e38.

[54] S. Schuerle, S. Pane, E. Pellicer, J. Sort, M. D. Baro, B. J. Nelson, Small 2012, 8, 1498.

[55] S. Tottori, L. Zhang, F. Qiu, K. K. Krawczyk, A. Franco-Obregon, B. J Nelson, Adv. Mater. 2012, 24, 811. 
a
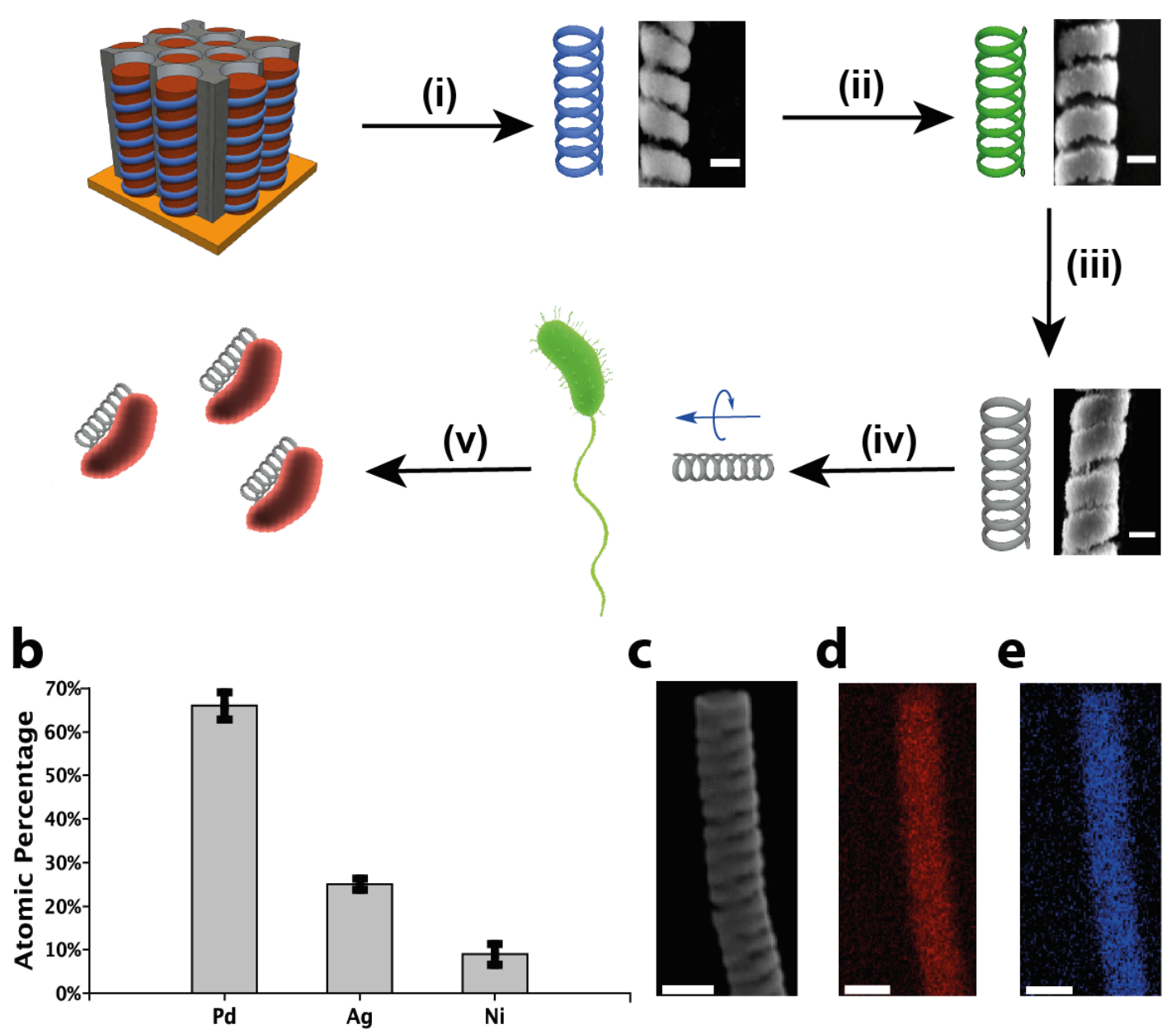

Figure 1. Fabrication overview of $\mathrm{Pd} / \mathrm{Ni} / \mathrm{Ag}$ nanocoil structures and their application for bacteria killing: a) (i) Electrodeposition of $\mathrm{Pd}$ (blue)/Cu (orange) nanowires in $\mathrm{AAO}$ templates (grey), followed by dissolution of the template $(\mathrm{NaOH})$ and selective de-alloying of $\mathrm{Cu}$ (HNO3). (ii) Physical vapor deposition of $7.5 \mathrm{~nm}$ Ni layer (green). (iii) Physical vapor deposition of $10 \mathrm{~nm} \mathrm{Ag} \mathrm{layer} \mathrm{(grey).} \mathrm{(iv)} \mathrm{Directed} \mathrm{locomotion} \mathrm{of} \mathrm{the} \mathrm{nanocoils} \mathrm{in} \mathrm{low}$ magnetic fields. (v) Antibacterial activity by direct contact killing. b) Characterization of metal distribution in atomic percentage (at\%) on the Pd/Ni/Ag nanocoils by EDX analysis. c) SEM image of a Pd/Ni/Ag nanostructure (scale bar is $200 \mathrm{~nm}$ ). d) EDX map of Pd distribution (scale bar is $200 \mathrm{~nm}$ ). e) EDX map of Ag distribution (scale bar is $200 \mathrm{~nm}$ ). 
WILEY-VCH
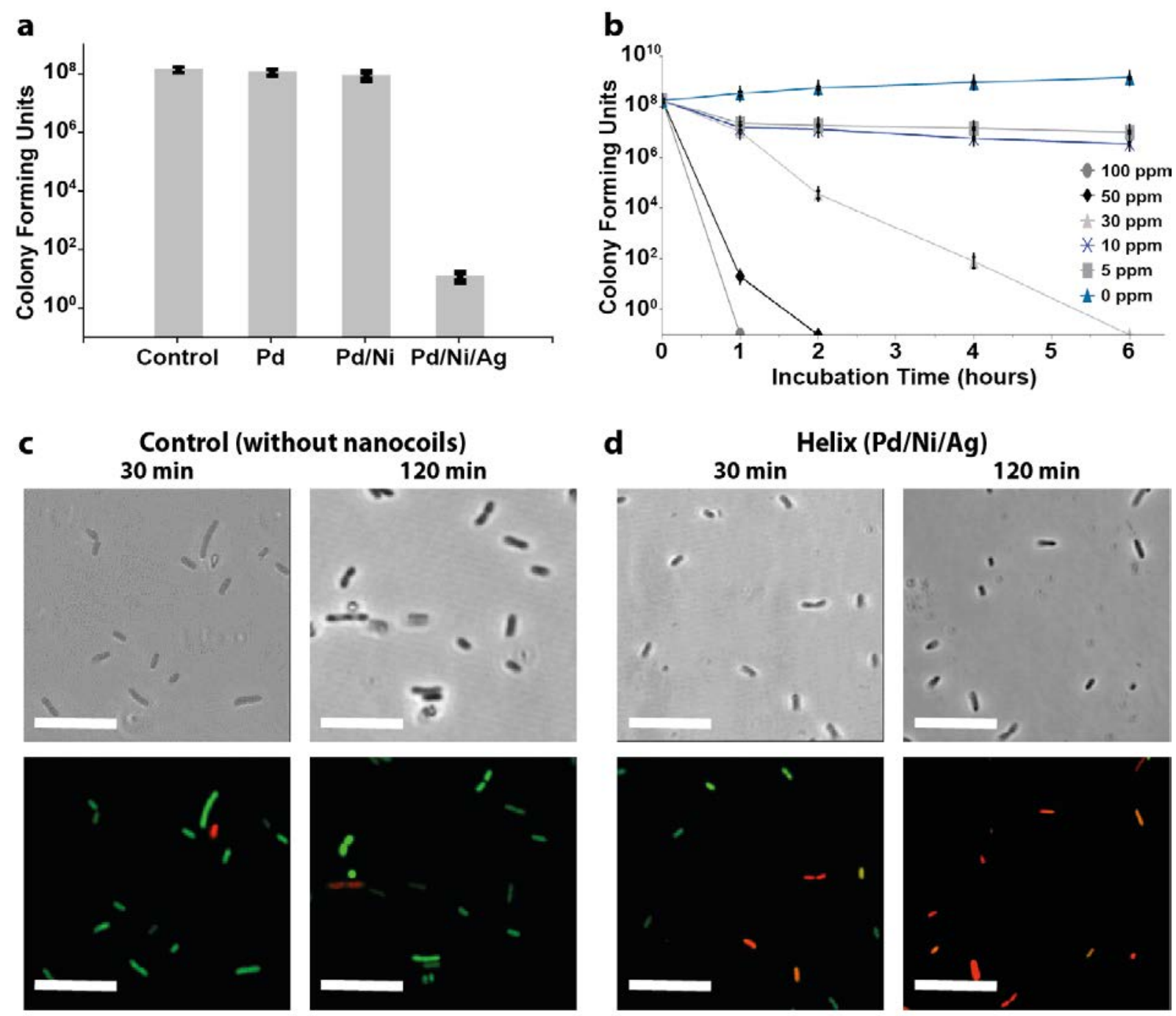

Figure 2. a) Antibacterial activity against E.coli XL-1 after 1 h exposure to nanocoils (50 ppm) from each fabrication step. b) Time and concentration dependency for E.coli killing by Pd/Ni/Ag nanocoils. c,d) CLSM images of E.coli incubated with 50 ppm by LIVE/DEAD BacLight bacterial viability assay. Bacterial cells with intact membrane are stained green, and bacteria with compromised membrane are stained orange or red (all scale bars are $10 \mu \mathrm{m}$ ). 


\section{WILEY-VCH}
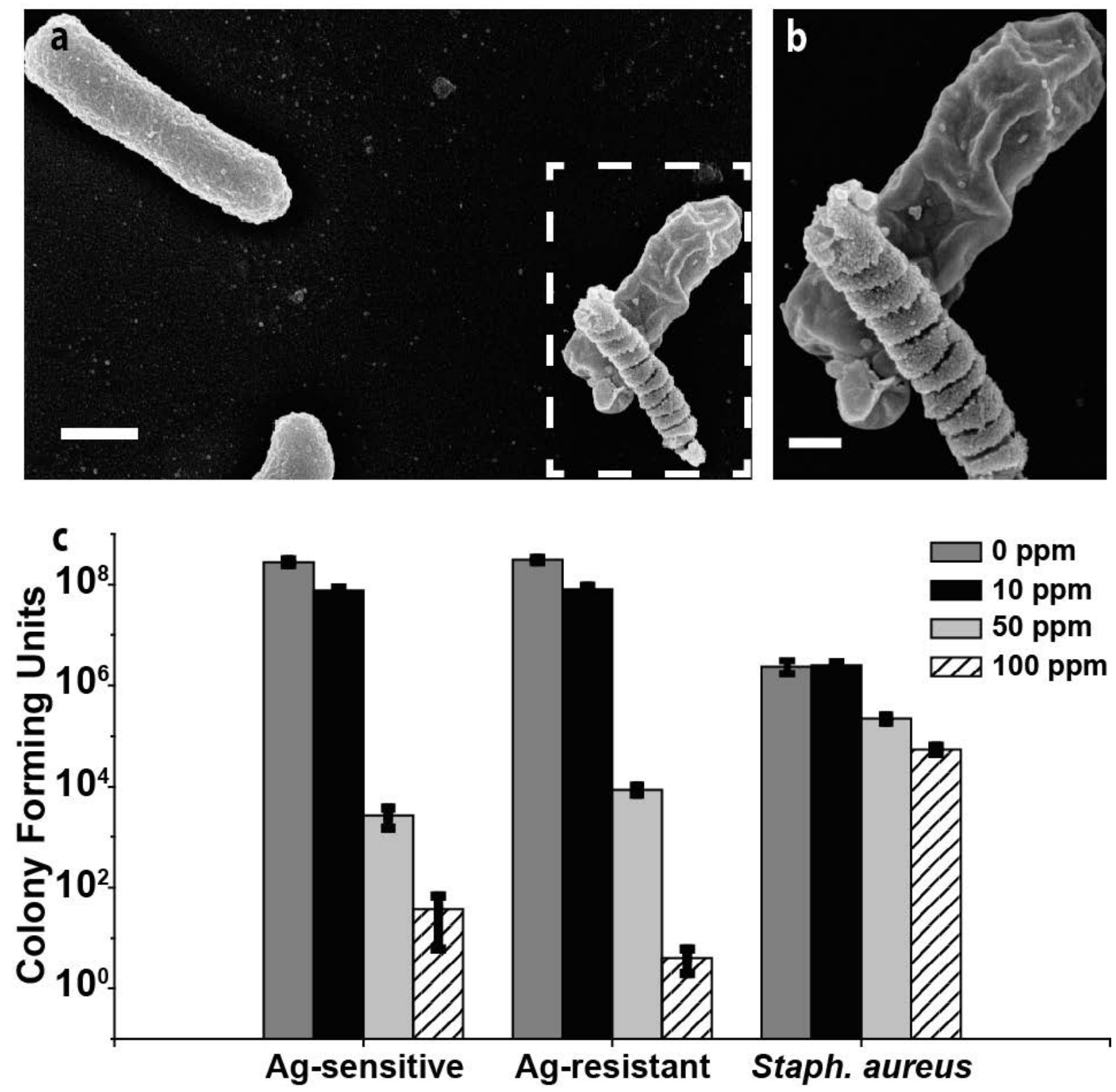

Figure 3. a) A SEM image showing morphological changes of the E. coli membrane in contact with the $\mathrm{Pd} / \mathrm{Ni} / \mathrm{Ag}$ nanocoil (scale bar is $500 \mathrm{~nm}$ ). b) Close-up SEM image of the $E$. coli bacterium with compromised membrane in contact with the Ag-coated nanocoil (Scale bar is $200 \mathrm{~nm}$ ). c) Antibacterial activity of different Pd/Ni/Ag nanocoil concentrations against Gram-negative Ag-ion sensitive and tolerant E.coli as well as Gram-positive MRSA. 


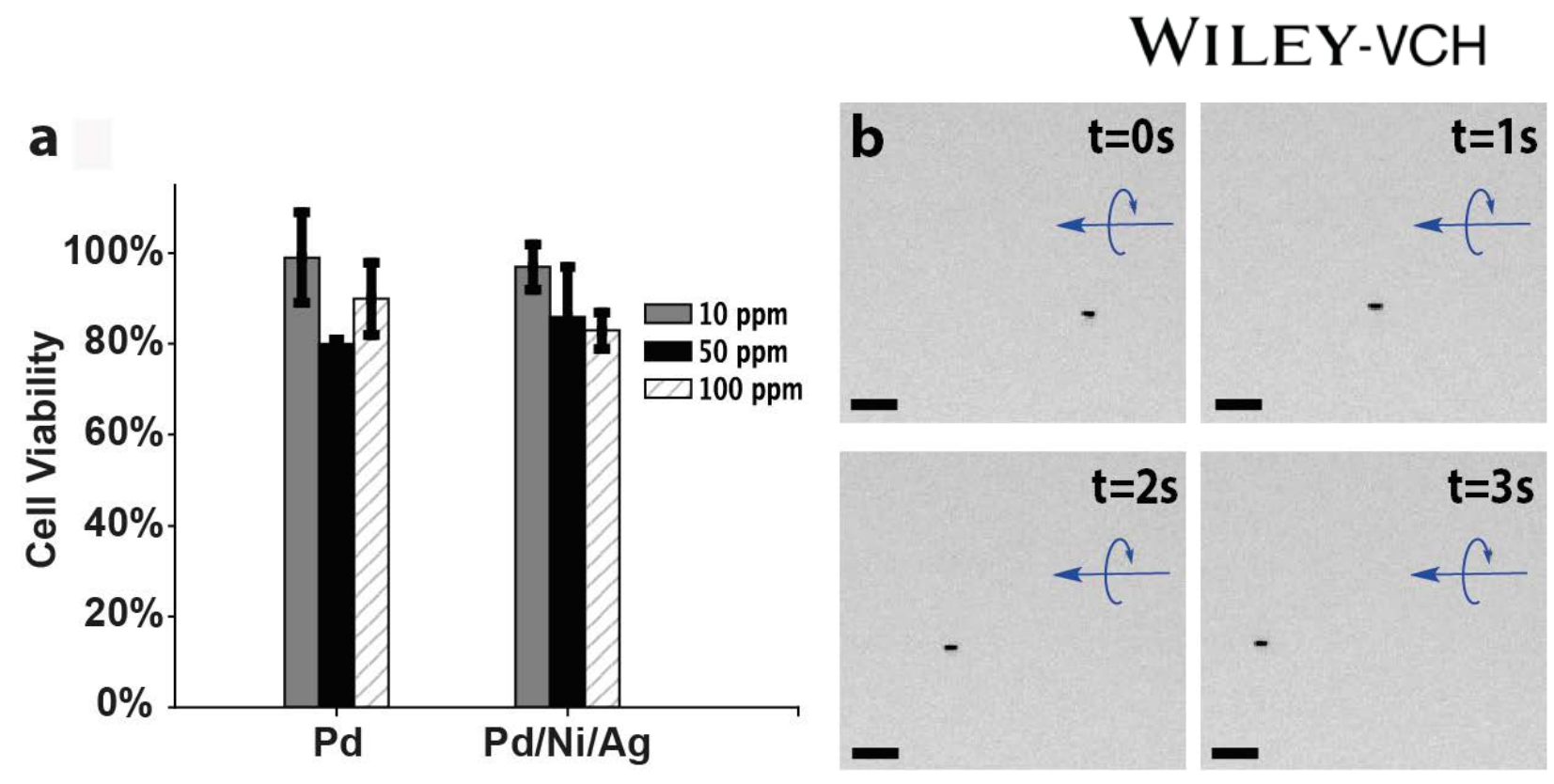

Figure 4. a) MTT cell viability assay to evaluate the toxicity of $\mathrm{Pd}$ and $\mathrm{Pd} / \mathrm{Ni} / \mathrm{Ag}$ nanocoils towards NIH-3T3 mouse fibroblasts at different concentrations. b) Time lapse images of a moving $\mathrm{Pd} / \mathrm{Ni} / \mathrm{Ag}$ nanocoil by rotating magnetic fields (all scale bars are $5 \mu \mathrm{m}$ ). 


\section{WILEY-VCH}

\section{Table of contents}

Antibacterial, silver-coated magnetic nanocoils show high killing efficiency against Gram-negative and Gram-positive bacteria by direct contact interaction. Low cytotoxic properties and precise magnetic locomotion make the proposed nanocoil an ideal candidate to combat multi-drug resistant bacteria in biomedical and environmental applications.

Keywords: Electrodeposition, nanocoil, magnetic, antibacterial effect, silver.

Authors: Marcus Hoop, Yang Shen, Xiang-Zhong Chen*, Fajer Mushtaq, Loredana M. Iuliano, Mahmut S. Sakar, Andrew Petruska, Martin J. Loessner, Bradley J. Nelson, and Salvador Pané*.

Title: Magnetically driven silver-coated nanocoils for efficient bacterial contact killing

TOC Figure:

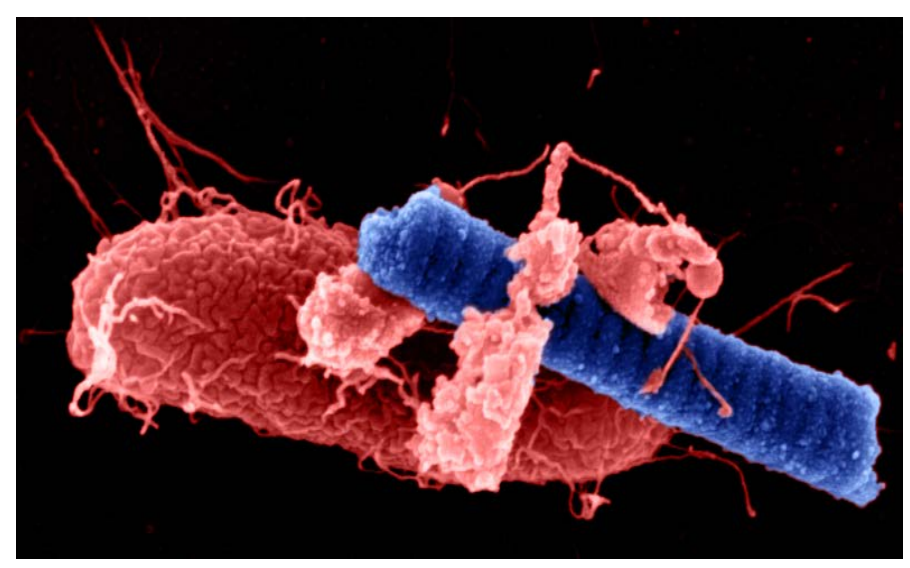

